\title{
AS REDES DE COOPERAÇÃO COMO UM MECANISMO PARA O APRIMORAMENTO DOS PROCESSOS DE CRIAÇÃO DO CONHECIMENTO: UM ESTUDO EXPLORATÓRIO NO SETOR CALÇADISTA
}

\section{THE COOPERATION NETWORKS AS A MECHANISM FOR THE IMPROVEMENT OF THE PROCESSES OF THE KNOWLEDGE CREATION: AN EXPLORATORY STUDY IN SECTION OF SHOES}

\author{
Lucyanno Moreira Cardoso de Holanda ${ }^{1}$; Isabela Assis Guedes ${ }^{2}$; Ana Cecília Feitosa de \\ Vasconcelos $^{3}$; Gesinaldo Ataíde Cândido ${ }^{4}$ \\ ${ }^{1}$ Universidade Federal de Campina Grande - UFCG - Campina Grande - PB, Brasil \\ lucyanno@gmail.com \\ ${ }^{2}$ Universidade Federal de Campina Grande - UFCG - Campina Grande - PB, Brasil \\ isabelarosas@gmail.com \\ ${ }^{3}$ Universidade Federal de Campina Grande - UFCG - Campina Grande - PB, Brasil \\ acvasconcelos@gmail.com \\ ${ }^{4}$ Universidade Federal de Campina Grande - UFCG - Campina Grande - PB, Brasil \\ gacandido@uol.com.br
}

\begin{abstract}
Resumo
O atual contexto organizacional é caracterizado pela mutação constante, concorrência cada vez mais acirrada e a necessidade das organizações buscarem os mecanismos e ações mais adequadas para o alcance de maior competitividade. Nesta perspectiva, o novo parâmetro para análise organizacional tem sido o conhecimento, o qual pode ser gerenciado, buscando a sua adequação para a estrutura, formas de funcionamento e as estratégias empresariais. Com este intuito, surgem vários conceitos e modelos facilitadores para as práticas da Gestão do Conhecimento que pode ser potencializado através de um contexto fisico, virtual e mental que é denominado pelos japoneses de ba. Nesses espaços o conhecimento é criado, compartilhado e utilizado, o que proporciona um aumento nos níveis de solicitude entres as pessoas. Partindo desse pressuposto, o artigo mostra a percepção dos gestores atuantes no setor calçadista em Campina Grande - PB acerca da sua atuação em redes de cooperação e a existência de ba organizacionais e seus benefícios, o que contribui para a criação de um ambiente de interação de aprendizado coletivo. $O$ estudo caracteriza-se como exploratório, com a utilização de técnicas qualitativas para coleta e análise dos dados. Os resultados apontam que a existência de características de redes de cooperação no setor tem contribuído para a formação e existência de ba organizacionais, de várias formas, com destaque para participação em feiras e exposições, viagens conjuntas; reuniões informais e ocorridas junto às instituições de apoio e utilização mais intensiva de recursos de tecnologia de informação e de comunicação.
\end{abstract}

Palavras-chave: gestão, cooperação, conhecimento. 


\section{Introdução}

O aumento da competitividade e o intenso desenvolvimento tecnológico têm imposto grandes desafios às organizações no sentido de rever constantemente suas formas de gestão e de adaptação ao ambiente no qual estão inseridas. Esse cenário se caracteriza pela necessidade de adoção da gestão capaz de antecipar-se às mudanças de mercado. A crescente preocupação com as mudanças que o mundo vem passando já faz parte do cotidiano das pessoas e das organizações, sinalizando a exigência de um esforço de todos no sentido de se transformarem em competidores mais capazes, a fim de enfrentar um ambiente de mercado cada vez mais mutante e desafiador.

Neste contexto, a busca da vantagem competitiva pelas organizações, por meio da valorização dos recursos humanos e da capacidade de gerenciamento do conhecimento, vem ocasionando o surgimento das organizações do conhecimento. A Gestão do Conhecimento despontou como uma nova sistemática de trabalho no ambiente organizacional. Esse fato conduz a uma série de mudanças organizacionais que possibilitam a criação, a disseminação e a utilização do conhecimento, almejando atingir os objetivos da organização.

Apesar da premência desta necessidade, as organizações, de um modo geral, não praticam a cultura do conhecimento, e muitas das que acreditam estarem trabalhando com essa ferramenta, limitam-se a contratar profissionais capacitados intelectualmente e colocá-los para trabalhar, sem se ater ao fato de que é necessário criar estruturas para a criação, a disseminação e a efetiva utilização do conhecimento. Neste caso, torna-se necessária uma mudança de cultura que permita e incentive as pessoas às aprendizagens individual e organizacional capazes de criar, utilizar e disseminar o conhecimento.

Diante desse novo panorama, a Gestão do Conhecimento (GC) surge como uma alternativa eficaz, permitindo às empresas melhoria de inovação nos processos, produtos e na gestão. Além disso, acarreta em um aumento nos níveis de solicitude entre as pessoas.

A principal problemática parte da perspectiva de quais são os meios que as organizações devem utilizar para conseguir "gerenciar" o conhecimento que é criado e potencializá-lo de forma que dele se crie inovações, sobretudo nas PME’s (pequenas e médias empresas).

Nonaka e Takeuchi (2002) enfatizam que as condições favoráveis para criação do conhecimento organizacional passam pelo processo denominado SECI (Socialização, Externalização, Combinação e Internalização) e necessitam de um ambiente físico, virtual e mental, os quais os japoneses denominam de $B a$. A partir dele o conhecimento é criado, compartilhado e utilizado.

Partindo dessa problemática, o artigo tenta demonstrar que, a partir da criação de redes de cooperação e dos $B a$ organizacionais em PME's, o conhecimento poderá ser potencializado, 
proporcionando um ambiente de aprendizado coletivo, sobretudo pela dinâmica de interação que ocorre entre as empresas.

Além desta parte inicial que contextualiza a Gestão do Conhecimento e a necessidade da criação do conhecimento, são abordados no referencial teórico os conceitos principais de Gestão do Conhecimento, das redes de cooperação, de $B a$ e de solicitude nas organizações. Em seguida são explicitados os procedimentos metodológicos para coleta e análise dos dados. Depois disso, são apresentados os resultados da pesquisa, nos quais são abordadas a caracterização do setor calçadista, aqui considerado como uma rede de cooperação, e as formas de existência e funcionamento de $B a$ no setor, o qual apresenta elementos capazes de aprimorar os processos de criação do conhecimento. Depois disso, são feitas as considerações finais, mencionando a necessidade da realização de outros estudos para confirmar base conceitual e conhecer como em outros contextos organizacionais são aplicados os mecanismos da Gestão do Conhecimento.

\section{Gestão do Conhecimento: principais conceitos}

O conceito e a prática da Gestão do Conhecimento não é algo novo, uma vez que sempre foi aplicado nas empresas de maneira informal. Vale salientar ainda que, dada a amplitude teórica do tema em pauta, existem variadas abordagens operacionalizadas pelos diversos autores explicitados no quadro abaixo:

Quadro 1 - Abordagens conceituais para Gestão do Conhecimento

\begin{tabular}{|c|c|}
\hline Autores & Abordagem \\
\hline Wiig (1993) & $\begin{array}{l}\text { Baseada nas práticas de exploração do conhecimento e sua adequação a } \\
\text { partir de práticas de gestão específicas. }\end{array}$ \\
\hline Leornard-Barton (1995) & $\begin{array}{l}\text { Foco em atividades que envolvem: 1) busca de soluções criativas, de } \\
\text { forma compartilhada; 2) implementação e integração de novas } \\
\text { metodologias e ferramentas nos processos atuais; 3) prática de } \\
\text { experimentos, a partir de protótipos e projetos piloto para } \\
\text { desenvolvimento de competências; 4) importação e absorção de } \\
\text { metodologias e tecnologias externas. }\end{array}$ \\
\hline Nonaka \& Takeuchi (1997) & $\begin{array}{l}\text { Baseada na transformação do conhecimento explícito em conhecimento } \\
\text { tácito e vice-versa, a partir das práticas de: combinação (explícito p/ } \\
\text { explícito); internalização (explícito p/ tácito); socialização (tácito p/ tácito) } \\
\text { e externalização (tácito p/ explícito). }\end{array}$ \\
\hline Barclay \& Murray (1997) & Ênfase em aspectos culturais e de redefinição de processos. \\
\hline Sveiby (1998) & $\begin{array}{l}\text { Orientada para a gestão da informação, para a aprendizagem } \\
\text { organizacional e para definição e utilização de competências. }\end{array}$ \\
\hline Edvinsson \& Malone (1998) & $\begin{array}{l}\text { Baseada em três tipos de recursos organizacionais e seus inter- } \\
\text { relacionamentos: capital humano; capital organizacional e capital do } \\
\text { cliente. Nesta abordagem, estas relações geram o capital intelectual de } \\
\text { uma organização e que, neste sentido, é preciso expandir a expertise, } \\
\text { encorajar a inovação e exercitar a integridade. }\end{array}$ \\
\hline
\end{tabular}

Fonte: Silva Filho e Cândido, (2003). 
Este estudo envolve a identificação das práticas organizacionais mais adequadas para a Gestão do Conhecimento e mais especificamente, capazes de criar e potencializar o uso mais intensivo de informações que redundem em práticas inovadoras. Essas práticas são facilitadas nas empresas que atuam sob a forma de redes de cooperação, nas quais existem maiores facilidades para o surgimento e consolidação dos $B a$ organizacionais, capazes de potencializar a ampliação interorganizacional do conhecimento.

Deste modo, optou-se pela abordagem de Nonaka e Takeuchi (1997), considerando que os autores exploram de forma mais específica os processos de criação do conhecimento, a partir de conceitos e suas múltiplas formas de aplicação no contexto organizacional.

\subsection{Redes de cooperação}

Com o reconhecimento da importância do ambiente, do contexto e de determinadas contingências, foi despertada nos anos 30 a utilização sistemática da idéia, dos conceitos e aplicações de Redes pelas mais variadas áreas do conhecimento, partindo do princípio de que o homem é um ser eminentemente social e necessita durante toda a sua existência manter diversos tipos de relacionamentos e interações com outras pessoas. Este tipo de comportamento social pode ser estendido para os grupos sociais, dentro do princípio de que estes grupos são formados por pessoas. Os princípios fundamentais da aplicação dos conceitos de Redes são: a interação, o relacionamento, a ajuda mútua, o compartilhamento, a integração e a complementaridade (Cândido 2001).

Nohria (1992) aponta três razões para a utilização do paradigma de Redes no ambiente organizacional, são elas: 1) a emergência de um novo padrão de competitividade faz com que as organizações busquem, ao invés de relações competitivas, relações colaborativas que as unam em redes de interligações laterais e horizontais, tanto interna quanto externamente; 2) os recentes desenvolvimentos no campo da tecnologia da informação têm proporcionado uma revolução de amplo escopo nos arranjos, operações e interligações das organizações em todo o mundo e 3) o amadurecimento da análise de redes como disciplina acadêmica.

No ambiente organizacional e dos negócios, a rede representa uma forma de organização intermediária entre a empresa e o mercado, redefinindo-se os princípios das estruturas burocráticas e da hierarquia inflexível pela necessidade premente de vinculação entre os processos referentes à coordenação da atividade econômica, surgindo daí vários tipos de rede, tais como as redes de inovação, as redes estratégicas, as redes flexíveis e multidirecionais de Pequenas e Médias Empresas. (Cândido 2001). 
Para as PME's o trabalho em rede facilita a sua manutenção no mercado e a obtenção de vantagens competitivas sustentáveis, devido ao maior fluxo de informações e conhecimentos compartilhados, assim como a viabilização de ações conjuntas com as empresas parceiras. Entretanto, para facilitar essa cooperação entre estas empresas, torna-se necessária a criação de um ambiente que proporcione tais interações emergentes. Nesse sentido, surge a definição e a utilização do $B a$, que estão explicitadas e evidenciadas no tópico a seguir.

\subsection{Conceituação de $b a$}

Nonaka e Konno (1998) definem $B a$ como um espaço compartilhado para as relações emergentes, podendo ser um espaço físico (um escritório, espaço de negócios (redes), etc.), virtual (um e-mail, uma teleconferência, etc.), mental (das experiências compartilhadas, das idéias, dos ideais) ou uma múltipla combinação destas, sendo considerado um espaço compartilhado que serve de base para a criação de conhecimento, seja este individual ou coletivo.

O que diferencia as empresas que utilizam o $B a$ das empresas que não o utilizam e dispõem apenas de interações normais humanas é o seu conceito de criação de conhecimento, uma vez que aquele não trata somente de simples interações entre os indivíduos, mas também disponibiliza uma plataforma para o conhecimento individual/coletivo avançado para a criação de conhecimento. Nonaka e Konno (1998) ainda propõem que o $B a$ é construído de “informação necessária” à criação de conhecimentos, tanto individuais quanto coletivos, sendo as interações, desse modo, condicionadas por esse contexto rico em conhecimentos. As trocas de dados, de informação, de opinião, de colaboração e de uma mobilização sobre um projeto, confrontado às necessidades e ao desconhecido convergem ao $B a$ dentro das organizações (Fayard 2003). Deste modo, o papel dos dirigentes e executivos dentro das organizações é disponibilizar o $B a$ para a criação de conhecimento; sua tarefa é gerenciar a emergência do conhecimento. O $B a$ não vem à realidade por decreto. Não é produzido pelo modelo do command and control próprio da gerência piramidal tradicional. Ao contrário, é ajustado por atores voluntários dentro de um ambiente energize and stimulate com atenção ao respeito mútuo. O $B a$ é fundamentalmente subjetivo e relacional, envolvendo os atores pelo fato de ser orientado pelo interesse e por não existirem fortes conflitos nos relacionamentos humanos (Fayard 2003). Nesse sentido, para que o $B a$ se torne efetivamente produtivo para as organizações (e entre elas), é necessário que haja a promoção de solicitude. 


\subsection{Solicitude nas organizações}

As empresas vivem em ambientes de hipercompetição, onde necessitam encontrar meios de obterem vantagens competitivas sobre seus concorrentes a todo o momento. Conseqüentemente, a alta gerência, os sistemas, as estratégias e a estrutura das empresas passam a seguir essa postura. Com efeito, os trabalhadores são afetados em seus sistemas de remuneração e posição, causando uma possível diminuição nos níveis de confiança e cooperação.

Uma das alternativas que possibilita bons níveis de solicitude entre as pessoas é a criação de redes de cooperação e dos $B a$ organizacionais. É necessário lembrar que a solicitude pode existir nas organizações sem a necessidade da criação de $B a$ ou de redes de cooperação, mas estes não podem prosperar sem a presença da solicitude, já que o conhecimento tácito das pessoas, base para todo conceito da criação, é suscetível às barreiras impostas pela sua ausência.

Para compartilhar o conhecimento pessoal, os indivíduos devem confiar no que os outros estejam dispostos a ouvir e a reagir às suas idéias (Von Krogh, 2001). Os bons relacionamentos diminuem a desconfiança, o medo e a insatisfação, proporcionando possibilidades de exploração de ambientes desconhecidos como novos mercados, novos produtos e novas tecnologias.

Segundo Sabel (1991), a confiança nunca poderá intencionalmente ser criada e sim gerada a partir de uma estrutura ou de contextos adequados. Já Perrow (1992) argumenta que, embora a confiança não possa ser criada, ela poderá ser encorajada por uma estrutura ou por um contexto que podem ser deliberadamente criados.

Portanto, a solicitude trata de relações de ajuda mútua ao aprendizado, contribuição de conscientização das oportunidades, além de suas conseqüências, e de fomentar conhecimentos pessoais e compartilhar seus insights.

Von Krogh et al (2001) divide, para fins de melhor compreensão, o conceito de solicitude em cinco dimensões: confiança mútua, empatia ativa, acesso à ajuda, leniência nos julgamentos e coragem.

- Confiança mútua é algo recíproco. Só se aceita ajuda quando se acredita nas boas intenções de quem oferece. As pessoas se apresentam por meio de histórias e daí vai se cultivando respeito, gerando garantias e reputação.

- Empatia ativa possibilita avaliação e compreensão das necessidades alheias. É a tentativa de pôr-se no lugar dos outros, de compreender a situação particular, seus interesses, habilidades, sucessos, fracassos, oportunidades e problemas.

- Acesso à ajuda, trata-se da manifestação de ajuda espontânea nas organizações. Os profissionais quanto mais adquirem conhecimentos, tem a "obrigação" de solidariedade para com os colegas e empresa. 
- Leniência nos julgamentos significa ter cautela em julgar as pessoas. Para a criação do conhecimento os julgadores devem analisar todas as variáveis necessárias para evitar tomar decisões erradas que podem prejudicar todas as outras cinco dimensões.

- Coragem desempenha papel importante em três aspectos. Primeiro, as pessoas devem ser corajosas para aceitar as boas idéias vindas de outras pessoas. Segundo, devem ter bravura para submeter seus conceitos a um processo de julgamento. E terceiro, oferecer feedback às pessoas que estiverem necessitadas.

Dentro de uma empresa os relacionamentos organizacionais podem existir em basicamente dois níveis - alta solicitude e baixa solicitude.

A alta solicitude é caracterizada por grande confiança, empatia ativa, acesso à ajuda, leniência no julgamento e coragem entre os membros. As pessoas criam conhecimento individual mediante transferência de insights e os grupos geram o conhecimento social por meio da convivência. No que se refere à baixa solicitude, tais comportamentos são quase ausentes. A criação do conhecimento individual ocorre por meio de captura, e boa parte do conhecimento social por meio de transação. A figura 1 resume esses processos.

Figura 1: Níveis de solicitude nos processos de criação do conhecimento

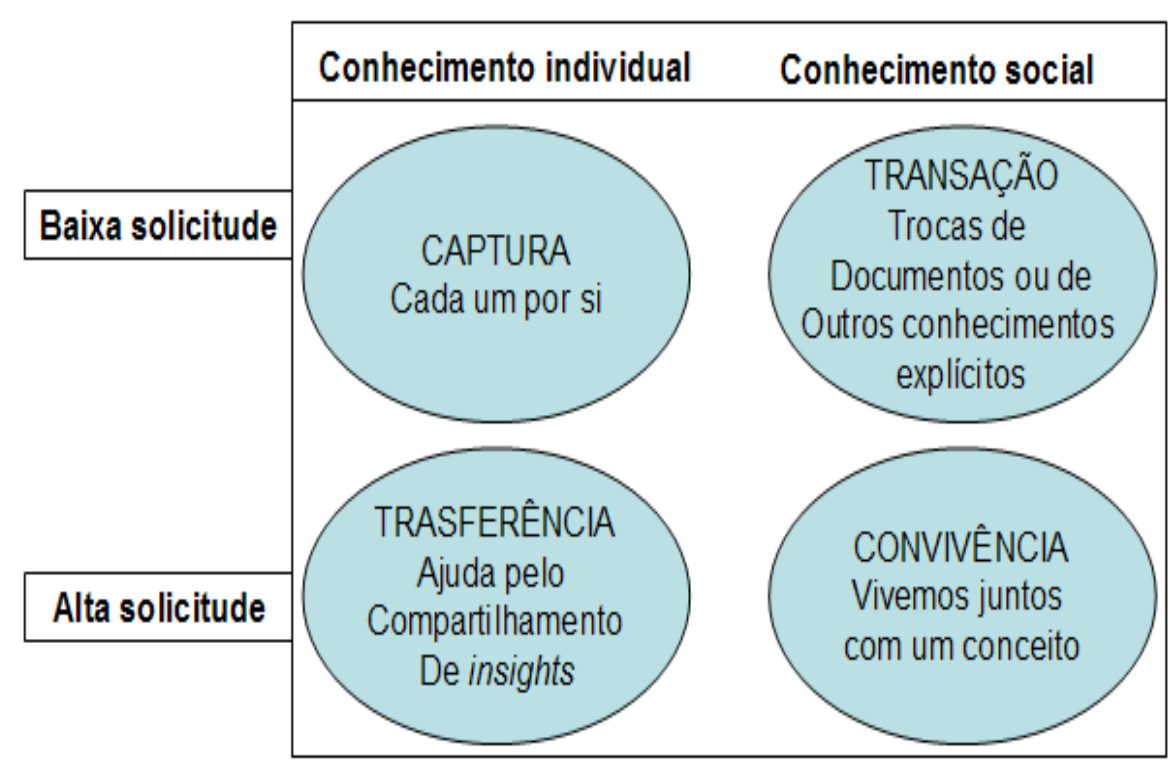

Fonte: Von Krogh e Nonaka (2001)

A partir do conteúdo aqui explicitado, pode-se inferir que a criação do conhecimento organizacional é um processo complexo que envolve um conjunto de variáveis tecnológicas, estruturais e principalmente de ordem sócio-comportamental, com implicações múltiplas nas formas de funcionamento da empresa e nas posturas e ações das pessoas. No entanto, esse processo pode ser facilitado quando as empresas atuam sob a forma de redes de cooperação, nas quais as 
condições para a criação do $B a$ são facilitadas, criando um ambiente capaz de disseminar e compartilhar a informação para todos os setores e pessoas que compõem a empresa, condição esta imprescindível para que as organizações encontrem as formas devidas para sobrevivência e desenvolvimento.

\section{Procedimentos metodológicos}

O método de pesquisa desenvolvido busca aliar as concepções teóricas relativas ao tema e as necessidades práticas da coleta de dados. A pesquisa realizada pode ser caracterizada como sendo um estudo exploratório, definido por Marconi e Lakatos (1990) como sendo o tipo de pesquisa em que, a partir de um problema formulado, familiariza o pesquisador com um ambiente, fato ou fenômeno para realização de futuras pesquisas para modificar ou clarificar conceitos. Para os fins desta pesquisa foram utilizados métodos quantitativos e qualitativos para analisar todas as interrelações entre as variáveis pesquisadas.

Em função dos objetivos propostos foram necessárias evidências empíricas primárias, principalmente qualitativas, sobre a atuação das empresas em redes de cooperação e a existência de ba nas empresas pesquisadas. Essas evidências empíricas foram obtidas a partir de fontes múltiplas: empresários, gerentes, empregados, técnicos ligados às instituições de apoio a atividade econômica, etc.

Tais fontes foram acessadas com base em técnicas variadas, dentre as quais: acesso, coleta, tratamento e análise de dados secundários referentes a relatórios de pesquisa realizado no setor calçadista de Campina Grande, especificamente: Lemos e Palhano (2000) e Aragão (2003). Além disso, foram utilizadas as técnicas da observação não participante, envolvendo visitas técnicas a um conjunto de empresas do setor.

A escolha das empresas a serem pesquisadas ocorreu a partir de dados secundários obtidos junto à Federação das Indústrias do Estado da Paraíba (FIEP), no qual são discriminadas as características das empresas do setor. Considerando que o conjunto das empresas que formam o setor calçadista na região é homogêneo, ou seja, não existem diferenças significativas entre elas em termos de perfil, formas de atuação e níveis de competitividade, para a definição da amostra da pesquisa optou-se pela amostragem não probabilística do tipo intencional. Neste sentido, a população é composta de 48 empresas, sendo que, deste total, foram escolhidas 12 empresas mais atuantes e com maior interação com as instituições de apoio a atividade econômica na região para a aplicação das técnicas de pesquisa propostas.

O tratamento dos dados foi realizado a partir de pesquisa bibliográfica selecionada, lida, fichada, discutida e consolidada, servindo este material como fonte principal da pesquisa. Para a análise dos dados foi utilizada a técnica de análise de conteúdo temático, procurando compreender 
as inferências dos empresários do setor calçadista acerca das vantagens de atuação em redes de cooperação e a existência de $b a$ organizacionais e suas respectivas contribuições para o melhor funcionamento das suas empresas.

\section{A formação do setor de calçados em Campina Grande}

O surgimento da atividade coureira de Campina Grande iniciou-se no ano de 1923, com a criação de Curtumes na cidade, fruto da iniciativa pioneira da família Motta, quando a cidade possuía cerca de 4000 habitantes (Kehrle, 2002). Sua produção destinava-se inicialmente à confecção de selas, arreios e rédeas para montarias, muito utilizadas no meio rural. A vocação histórica para trabalhar com o couro e subseqüentemente com calçados e afins aparece como explicação predominante do desenvolvimento do arranjo produtivo coureiro-calçadista de Campina Grande. A produção do couro na região teve seu período de apogeu entre 1940-1954, sobretudo na Segunda Grande Guerra, quando exportavam seus produtos.

Apesar do seu declínio nos anos 70, Campina Grande ainda mostrou-se ser de relevada importância para o setor. No entanto, a sua importância veio a ser diminuída a partir dos anos 80 , fato ocorrido principalmente devido às dificuldades de funcionamento dos curtumes instalados na região.

O arranjo coureiro-calçadista de Campina Grande até meados da década de 1990 apresentava um quadro de desenvolvimento econômico promissor, com reflexos no município e na região (Pinhanez, 1998). Sua importância pode ser reconhecida a partir de alguns fatores como: i) a articulação de organismos públicos privados objetivando estimular o seu desenvolvimento através de estudos e diagnósticos; ii) A criação de uma Universidade Federal na década de 70 para formação de graduação e especialistas; iii) criação de um centro tecnológico de couro e calçado (CTCC), sendo este o único do nordeste; iv) o pioneirismo na atração de incentivos de grandes empresas vindas de outras regiões do país como fornecedores de insumos.

Hoje temos como uma herança histórica de produção de calçados de Campina Grande um vasto contingente de sapateiros e produtores informais, onde muitos adquiriram experiência na produção de calçados passando-se, de forma geral, em processo de difusão familiar de conhecimentos. $\mathrm{O}$ arranjo é composto fundamentalmente por produtores locais de micro, pequeno a médio porte de calçados e artefatos de couro ou de material sintético, sendo grande parte das empresas de estrutura familiar.

\section{Resultados - Utilização de ba e redes de cooperação para o aprimoramento dos processos de criação do conhecimento}


O estudo sobre o arranjo produtivo local de calçados em Campina Grande - PB possibilita a utilização de redes de cooperação e $B a$ nas suas empresas no que diz respeito à criação do conhecimento. As evidências empíricas observadas no decorrer do estudo serão analisadas e refletidas para compreensão sobre as relações entre a criação de conhecimento, $B a$ e redes de cooperação.

Os estudos mostram que existem vários $B a$ (físico, mental e virtual) que facilitam o processo de criação do conhecimento. Um desses espaços, sobretudo para a comunicação de conhecimento tácito, acontece através de visitas a feiras nacionais e internacionais. Por meio dessas visitas os empresários identificam possibilidades de melhoria nos processos, produtos e gestão, que geram ganhos substanciais para as empresas do arranjo.

A maior percepção sobre uso dessas ferramentas de criação do conhecimento são os ganhos e o aproveitamento de informações e conhecimento, sobretudo o tácito, entre as empresas. Nas reuniões entre os principais empresários do setor, as quais ocorrem periodicamente, observa-se um espaço de compartilhamento do conhecimento $(B a)$. A reunião funciona como espaço formal para o processo de decisões conjuntas, e os principais temas de discussão são: preços, viagens, feiras, concorrentes, compras conjuntas, representantes, novos fornecedores, novas tecnologias de produção e novas matérias-primas. Alguns empresários conversam informalmente com os demais do arranjo, tanto antes quanto após as reuniões, propiciando o compartilhamento de experiências e conhecimentos, como forma de buscar soluções e/ou alternativas que viabilizem o bom desempenho de suas empresas.

As viagens, visitas e exposições de produtos em feiras permitem aos empresários do arranjo a oportunidade de conhecer outras realidades e refletir conjuntamente sobre oportunidades, tendências e desafios. Esse conhecimento do mercado poderá se tornar um diferencial no momento de elaborar suas estratégias de custos, marketing e desenvolvimento de novos produtos.

Outro exemplo de espaço de criação do conhecimento está na utilização de recursos eletrônicos - telefone, e-mail e fax. O uso dessas tecnologias apresenta um papel importante para a comunicação e sistematização de conhecimento explícito. No arranjo produtivo de calçados o telefone e o fax são utilizados com maior freqüência, enquanto que as outras tecnologias de informação e comunicação (TIC's) são relativamente menos utilizadas. Essa evidência representa uma deficiência do arranjo na sua dinâmica de criação do conhecimento.

Com a oferta de cursos e palestras pelas instituições Senai, Parque Tecnológico, e de empresas de consultoria, abre-se um espaço para a troca de informações entre os empresários e entre estes e as instituições.

Por ser um setor tradicional, a vocação, aprendizado e experiência são transmitidos de geração para geração, caracterizando um $B a$ mental. 
Deste modo, um efetivo processo de criação do conhecimento, representado pelos diversos tipos de $B a$, proporciona a emergência de ativos de conhecimentos essenciais para criação de valor e de diferencial competitivo para as empresas.

\title{
5. Considerações finais
}

O presente estudo teve como principal objetivo buscar a compreensão da dinâmica de criação e utilização do conhecimento. A perspectiva de análise foi referente ao contexto ou espaço físico, virtual e mental, dentro do qual o conhecimento é criado e utilizado e é denominado pela expressão japonesa de $B a$. Partindo do princípio das dificuldades de criação do conhecimento, torna-se necessária a identificação dos meios que as organizações devem utilizar para conseguir "gerenciar" o conhecimento que é criado e potencializá-lo de forma que dele se crie inovações. Em função disto, a formação de redes de cooperações e dos $B a$ organizacionais constituem-se em mecanismos capazes de potencializar a ampliação interorganizacional do conhecimento.

Os resultados demonstram que a configuração em redes de cooperação proporciona às empresas do APL condições favoráveis a uma dinâmica ativa aos processos de criação e ampliação de seus conhecimentos. As redes de cooperação proporcionam uma comunidade de conhecimento que faz emergir vários tipos de $B a$ que dificilmente seriam encontrados em pequenas e médias empresas que atuam de forma isolada. O surgimento de alguns espaços formais e informais proporciona aos empresários possibilidades de compartilhamento de suas habilidades, experiências, emoções e know-how, devido a comunicação face a face e compartilhamento e disseminação de conhecimento tácito, sendo que as características desse tipo de conhecimento são consideradas essenciais para a sustentabilidade das vantagens competitivas.

Deve-se destacar que o conceito de $B a$ é de relevante importância para se entender a verdadeira natureza do processo de criação de conhecimento junto às organizações japonesas. No entanto, as teorizações e evidências apresentadas não têm a pretensão de ser uma teoria definitiva e sim visam estimular o debate e a crítica sobre os conceitos de criação de conhecimento e $B a$ junto à comunidade acadêmica brasileira.

\begin{abstract}
The current organizational context is characterized by the constant change, competition growing and the need of the organizations look for the mechanisms more appropriate actions for the reach of the competitiveness. In this perspective, the new parameter for organizational analysis has been the knowledge, which can be managed, looking for adaptation for the structure, operation forms and the business strategies. With this intention, several concepts and facilitative models appear for the practices of the Knowledge Management that it can be to get better through a context physical, virtual and mental that it is denominated by $b a$ Japanese. In those spaces the knowledge is created, shared and used, providing an increase in the care levels enter the people. Leaving of that
\end{abstract}


presupposition, the article shows the active managers' perception in the section of shoes in Campina Grande - PB concerning performance in cooperation nets and the organizational $b a$ existence and their benefits, what contributes to the creation of an atmosphere of interaction of collective learning. The study is characterized as exploratory, with the use of qualitative techniques for collection and analysis of the data. The results point that the existence of characteristics of cooperation nets in the section has been contributing to the formation and organizational $b a$ existence, in several ways, with prominence for participation in fairs and exhibitions, united trips; informal meetings and happened the support institutions and more intensive use of resources of technology of information close to and of communication.

Keywords: management; cooperation; knowledge.

\section{Referências}

ALBUQUERQUE, E. (1998) Patentes de invenção de residentes no Brasil (1980-1995): uma investigação sobre o papel dos direitos de propriedade intelectual na construção de um sistema nacional de inovação (Tese de Doutorado). Rio de Janeiro: Instituto de Economia da UFRJ.

ARAgÃo, P. O. R. de. Arranjos Produtivos de Couros e Calçados da Paraíba: Arranjo Produtivo de Couros e Calçados de Campina Grande/PB. João Pessoa, Relatório de Pesquisa: PROMOS/SEBRAE, 2004.

BARCLAY, R. U. e MURRAY, P. What is Knowledge Management. In: A Knowledge Praxis. 1997.

CÂNDIDO, G. A., ABREU, A. F. Os conceitos de redes e as relações interorganizacionais: um estudo exploratório. In: ENANPAD, 24, 2000. Florianópolis. Anais... Florianópolis: ANPAD, 2000. 1 CD.

CÂNDIDO, G.A., Fatores Críticos de Sucesso no Processo de Formação, Desenvolvimento e Manutenção de Redes Interempresariais do tipo Agrupamento Industrial entre Pequenas e Médias Empresas: Um Estudo Comparativo de Experiências Brasileiras. 2001, 356 f. Tese (Doutorado em Engenharia de Produção) - UFSCPPGEP Florianópolis - SC.

EDVINSSON, L. e MALONE, M. S. Capital Intelectual: descobrindo o valor real de sua empresa pela identificação de seus valores internos. São Paulo: Makron Books do Brasil, 1998.

FAYARD, P., Le concept de «ba» dans la voie japonaise de la création du savoir, Rapports d'Ambassade, Ambassade de France au Japon, Juin 2003.

KEHRLE, L.R. A indústria calçadista de Campina Grande - um estudo do setor industrial local, Relatório de Pesquisa, Recife - IPSA, 2002.

LEMOS, C.; PAlhano, A. Arranjo Produtivo Coureiro-Calçadista de Campina Grande/PB. Nota Técnica 22. Rio de Janeiro: IE/UFRJ, 2000.

LEONARD-BARTON, D. Wellsprings of Knowledge. Boston: Harvard Business School Press, 1995.

MARCONI, M.A. \& LAKATOS, E.M. Técnicas de pesquisa: planejamento e execução de pesquisas, amostragens e técnicas de pesquisa, elaboração, análise e interpretação de dados. 2a ed. São Paulo: Atlas, 1990.

NOHRIA, N. Is a network perspective a useful way of studying organizations? In: NOHRIA, N.; ECCLES, R. G. (Ed.). Networks and organizations: structure, form, and action. Boston: Harvard Business School Press, 1992. p. 1-22.

NONAKA, I.; TAKEUCHI, H., The knowledge-creating company: how Japanese companies create the dynamics of innovation. New York: Oxford University Press, 1997.

NONAKA, I., KONNO, N., The Concept of "Ba": Building a Foundation for Knowledge Creation, California Management Review, vol 40, n o. 3, spring, 1998.

PERROW, C. Small-Firm Networks. In: Nohria, N.; Eccles, R. Networks and organizations: structure, form and action. Boston: Harvard Business School Press, 1992. 
PINHANEZ, M. Training and Social Liaisons: Long - Lasting Industrialization in Northeast Brazil's shoe industrial. Firt year doctoral research paper. International Developement and Regional Planning Group - Department of urban studies na planning - Masachussetts Institute of Technological, Cambridge, MA, May 1998.

SABEL, C. Moebius-strip organizations and open labor markets: some consequences of the reintegration of conception and execution in a volatile economy. In: Coleman, J.; Bourdieu, P. Social theory for a changing society. Boulder: Westview Press, 1991.

SILVA, C. A. V. Redes de Cooperação de Micro e Pequenas Empresas: Um estudo das atividades de logística no setor metalúrgico de Sertãozinho - SP, São Carlos, 2004. Dissertação Mestrado.

SVEIBY, K. E. A Nova Riqueza das Organizações: gerenciando e avaliando patrimônios de conhecimento. Rio de Janeiro: Campus, 1998.

VON KROGH, G.; ICHIJO, K.; NONAKA, I. Facilitando a criação do conhecimento: reinventando a empresa com o poder da inovação contínua. Rio de Janeiro: Campus, 2001.

WIIG, K.M. Knowledge Management Foundations: thinking about-how people and organizations create, represent, and use knowledge. Arlington, Texas: Schema Press, 1993.

\section{Dados completos de todos os autores:}

\section{Nome completo: Lucyanno Moreira Cardoso de Holanda}

Filiação institucional: Universidade Federal de Campina Grande - UFCG

Departamento: Unidade Acadêmica de Administração e Contabilidade - UAAC

Função ou cargo ocupado: Estudante/Pesquisador

Endereço completo para correspondência (bairro, cidade, estado, país e CEP): R: Antonio Joaquim

Pequeno, 525/201 - Bodocongó, Campina Grande - PB, Brasil CEP: 58109-085

Telefones para contato:(83) 3333-2882 ramal 216

E-mail:lucyanno@gmail.com

\section{Nome completo: Isabela Assis Guedes}

Filiação institucional: Universidade Federal de Campina Grande - UFCG

Departamento: Unidade Acadêmica de Administração e Contabilidade - UAAC

Função ou cargo ocupado: Estudante/Pesquisada

Endereço completo para correspondência (bairro, cidade, estado, país e CEP): R: Maria de Lourdes

Crispim Lima, 460/202 - Bodocongó, Campina Grande - PB, Brasil CEP: 58109-095

Telefones para contato:(83) 3333-4877

E-mail:isabelarosas@gmail.com

\section{Nome completo: Ana Cecilia Feitosa de Vasconcelos}

Filiação institucional: Universidade Federal de Campina Grande - UFCG

Departamento:Unidade Acadêmica de Administração e Contabilidade - UACC

Função ou cargo ocupado: Estudante/Pesquisadora 
Endereço completo para correspondência (bairro, cidade, estado, país e CEP): R: Frei Martinho, 113, Conceição, Campina Grande, PB, CEP: 58102-433, BRASIL

Telefones para contato: (83) 8825-4990

E-mail:acvasconcelos@gmail.com

\section{Nome completo: Gesinaldo Ataíde Cãndido}

Filiação institucional: Universidade Federal de Campina Grande - UFCG

Departamento: Unidade Acadêmica de Administração e Contabilidade - UAAC

Função ou cargo ocupado: professor titular em Administração Geral

Endereço completo para correspondência (bairro, cidade, estado, país e CEP): Rua Esperidião

Rosas, 235/703, Expedicionários, João Pessoa-PB, CEP: 58041-070

Telefones para contato: (83) 33101217

E-mail: gacandido@uol.com.br

Recebido para publicação em: 06/07/06

Aceito para publicação em: 08/09/06 